\section{MRS Bulletin Volume Organizers guide technical theme topics for 2022}

\author{
mrs.org/bulletin
}

$\mathrm{T}$ he 2022 MRS Bulletin Volume Organizers, appointed by incoming MRS President Cherie R. Kagan, will guide the development of theme topics for the 2022 volume year. They are Giulia Galli (The University of Chicago, USA), Andrew M. Minor (University of California, Berkeley, USA), Chris Palmstrøm (University of California, Santa Barbara, USA), and Matthias Wuttig (RWTH Aachen University, Germany).

Giulia Galli is the Liew Family Professor of Electronic Structure and Simulations in the Pritzker School of

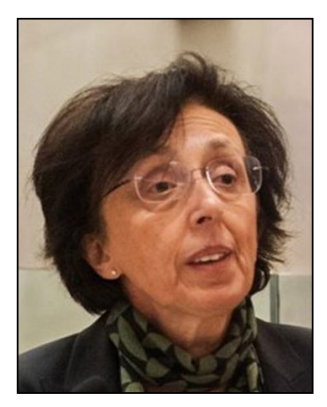

Molecular Engineering and a professor of chemistry at the University of Chicago. She also holds a senior scientist position at Argonne National Laboratory, where she is the director of the Midwest Integrated Center for Computational Materials. She earned a PhD degree in physics from the International School of Advanced Studies in Italy. She is a Fellow of the American Physical Society (APS), American Association for the Advancement of Science, and an elected member of the American Academy of Arts \& Science and the National Academy of Sciences. She is the recipient of numerous awards, including the Lawrence Livermore National Laboratory Science and Technology Award, the US Department of Energy Award of Excellence, the Materials Research Society Materials Theory Award, the APS David Adler Lectureship Award in Materials Physics, the Feynman Nanotechnology Prize in
Theory, the Medal of the Schola Physica Romana, and the Tomassoni-Chisesi Prize from the Sapienza University of Rome. Her research focuses on the development and use of theoretical and computational methods to understand and predict the properties and behavior of materials from first principles.

Andrew M. Minor is a professor at the University of California, Berkeley Materials Science and Engineering and holds a joint appointment at the Law-

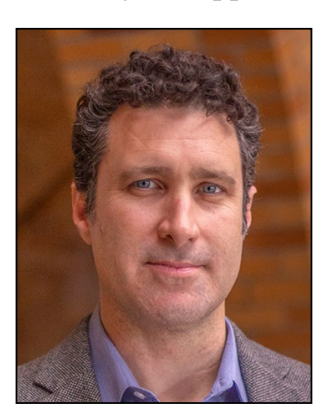

rence Berkeley National Laboratory, where he is the facility director of the National Center for Electron Microscopy in the Molecular Foundry. He received a $\mathrm{BA}$

degree in economics and mechanical engineering from Yale University and his MS and $\mathrm{PhD}$ degrees in materials science and engineering from UC Berkeley. He has co-authored more than 210 publications and presented over 130 invited talks on topics such as nanomechanics, lightweight alloy development, characterization of soft materials, and in situ TEM technique development. Minor's research interests lie at the intersection of advanced electron microscopy and materials science. His group focuses on the development of new and often in situ electron microscopy techniques to image and quantify nanoscale phenomena critical for the understanding of structure-property relationships in materials. His honors include the Lawrence Berkeley Labostanding Performance Award (2006 and (UC Berkeley) in the Department of ratory Materials Science Division Out-
2010), the American Institute of Mining, Metallurgical, and Petroleum Engineers Robert Lansing Hardy Award from The Minerals, Metals \& Materials Society (2012), and the Burton Medal from the Microscopy Society of America (2015).

Chris Palmstrom is a professor in electrical and computer engineering in the Materials Department at the University of California, Santa Barbara. His research involves atomic-level control and interface formation during molecular beam

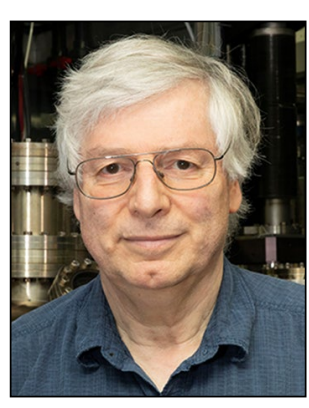
and chemical beam epitaxial growth of metallic compounds, metal oxides, and compound semiconductors. $\mathrm{He}$ received his $\mathrm{BSc}$ degree in physics and electronic engineering and a $\mathrm{PhD}$ degree in electrical and electronic engineering from the University of Leeds. After serving as a lecturer in Norway and a research associate at Cornell University, he joined Bellcore as a member of the Technical Staff in 1985. From 1994 to 2007, he was a Professor in the Department of Chemical Engineering and Materials Science at the University of Minnesota. In 2007, he joined the faculty at the University of California, Santa Barbara. He has pioneered dissimilar materials epitaxial growth studies using a combination of molecular beam epitaxial growth with in situ surface science probes and ex situ structural and electronic characterization. In 2015, he received the North American MBE Innovator Award and was named a US Department of Defense National Security Science and Engineering Faculty Fellow. In 2018, he received the David Adler Lectureship Award in the Field of Materials Physics.

Matthias Wuttig received his $\mathrm{PhD}$ degree in physics in 1988 from RWTH Aachen University/Forschungszentrum Jülich. From 1995 to 1997, he worked at Bell Labs in Murray Hill, N.J. He was a visiting professor at several institutions, including Lawrence Berkeley 


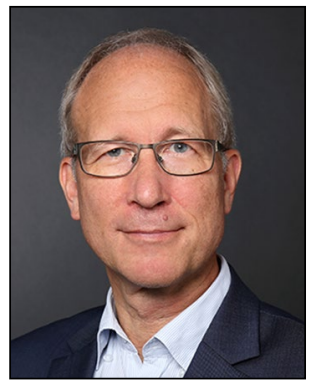

Laboratory, Stanford University, Zhejiang University, IBM Almaden Research Center, Data Storage Institute in Singapore, Centre Interdisciplinaire de
Nanoscience de Marseille, France, and the Chinese Academy of Sciences in Shanghai. In 1997, he was appointed full professor at RWTH Aachen University, where his work focuses on the design of novel functional materials. Since 2011, he heads a Collaborative Research Centre on resistively switching chalcogenides funded by the German National Science Foundation. In 2013, he received a European Research
Council Advanced Grant to realize novel functionalities by disorder control. In 2018, he became a Fellow of RWTH, and in 2019, he was selected as a MRS Fellow for "path-breaking contributions to the advancement of phase-change materials." Wuttig is a member of the German National Academy of Science and Engineering and has approximately 370 scientific publications.

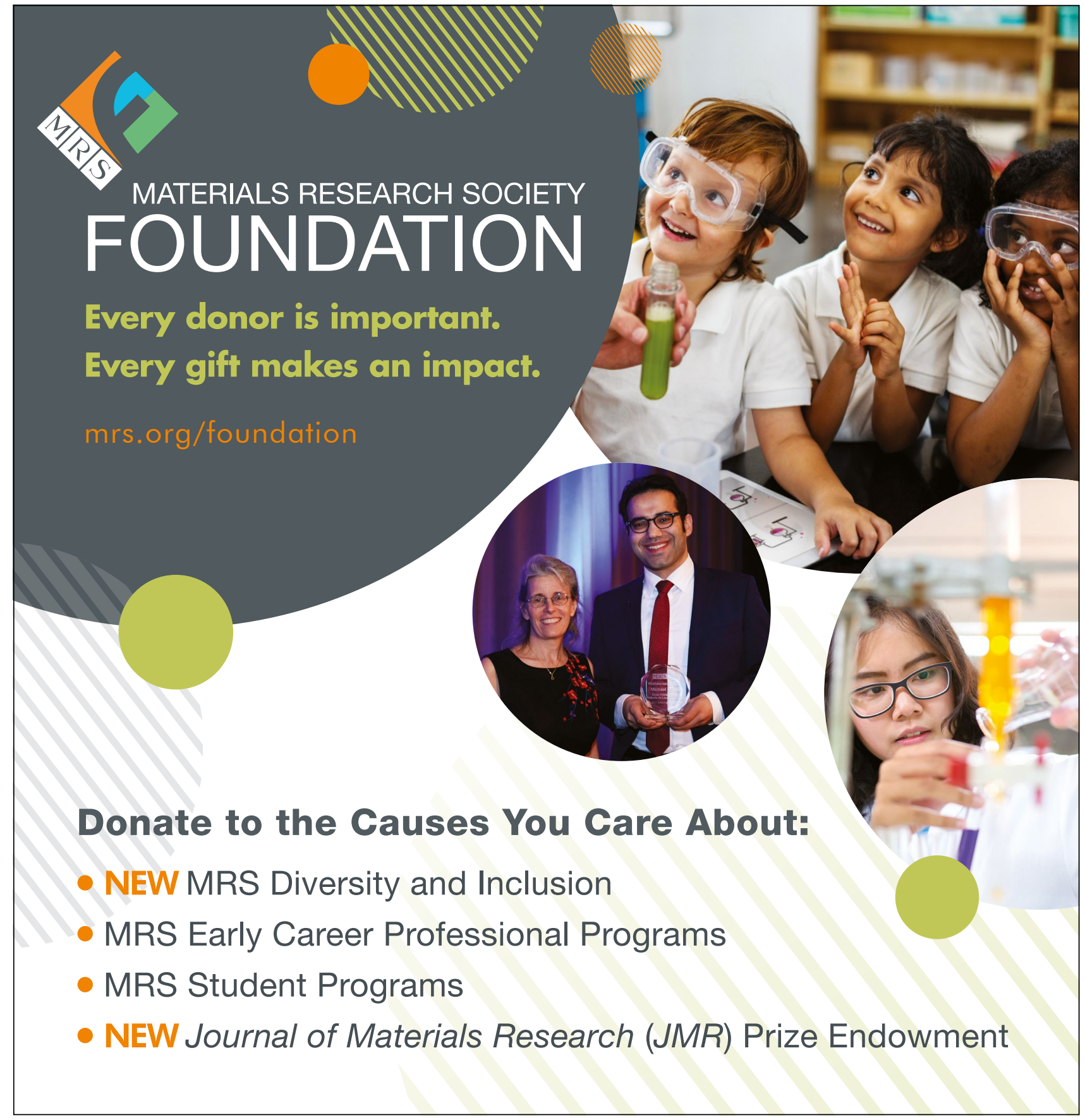

